\title{
Hacia un espacio europeo de aprendizaje para toda la vida. Evolución y desarrollo de la Educación Permanente en la Unión Europea
}

\author{
Fernando López Noguero \\ Universidad Pablo de Olavide
}

\section{Resumen}

En sus inicios, la Unión Europea (UE) concedió una mínima importancia a la educación en el marco de los derechos y las políticas sociales europeas, aumentando su peso paulatinamente, como comprobaremos en la propia evolución de la Educación Permanente en la UE, desarrollo que hemos dividido en cuatro etapas fundamentales.

Así, desde la proclamación del Año Europeo de la Educación y la Formación Permanentes en 1996, en el que se fomentó en el seno de la UE el concepto de aprendizaje durante toda la vida, la Educación Permanente ha cambiado radicalmente en Europa hacia una nueva realidad que se ha dado en llamar "Espacio Europeo de Aprendizaje para toda la vida", que analizamos en profundidad a través del análisis de la legislación y programas educativos existentes en la UE.

Palabras clave: Educación Permanente, Año europeo, Programas europeos, Dimensión europea, Espacio europeo, Aprendizaje para toda la vida.

\section{Summary}

In its early years, the European Union (EU) granted a minimal importance to education in the framework of rights and social policies in Europe, increasing its weight gradually, as we will check on the evolution of Continuing Education in EU development which we have divided into four basic stages.

So we will check how, since the proclamation of the European Year of Education and Training Standing in 1996, which fostered within the EU the concept of lifelong learning, Continuing Education has changed dramatically in Europe towards a new reality that has been termed a "European Area Learning for Life" which analyzed in depth through the analysis of legislation and educational programs existing in the EU.

KeYWoRDS: Continuing Education, European Year, European Programmes, European dimension, European Area, life long learning. 


\section{La eclosión de la Educación Permanente en un nuevo contexto social europeo}

La Educación Permanente se ha convertido en un tema central del debate político en la Unión Europea (UE) y constituido en un elemento clave en la reforma de los fondos estructurales y de las políticas comunitarias. Sin duda alguna, la educación constituye hoy día, un elemento crucial para la construcción y consolidación de una Europa unida, competitiva, solidaria y abierta al mundo.

En este sentido, el contexto mundial y europeo de principios de milenio está sufriendo un proceso de modernización aceleradísimo en multitud de ámbitos (cultural, tecnológico, económico, relacional, comunicacional, etc.), por lo que se hace necesaria, y ahora más que nunca, una acomodación constante por parte de la educación a estos procesos de cambio, a fin de que pueda responder a las nuevas demandas que van surgiendo en la sociedad.

El mundo está atravesando un periodo de transición y cambios profundos, y la UE no es ajena a los mismos. De hecho, todo parece indicar que la sociedad europea está a punto de entrar en una nueva era, mucho más imprevista y cambiante que las precedentes. Sin embargo, este periodo de transformación que sufrimos también es una oportunidad histórica para la Unión, ya que los tiempos de cambios son los más propicios para emprender reformas profundas.

Los ciudadanos tienen nuevas y enormes oportunidades de comunicación, desplazamiento y empleo, pero para poder aprovechar esas oportunidades y tomar parte activa en la sociedad que les ha tocado vivir, es fundamental adquirir continuamente nuevos conocimientos y aptitudes, ya que el creciente ritmo de evolución de la sociedad actual hace que cada vez sea necesaria una mayor preparación y cualificación para desarrollar con suficiencia un papel integrado en la sociedad en todos los ámbitos de la vida (Ríos, 2004).
Por todo ello, podríamos afirmar que la Unión, en transición hacia un nuevo modelo, se encuentra ante un auténtico reto de supervivencia, donde el conocimiento, las cualificaciones y las capacidades de aprendizaje se deben adaptar a los nuevos tiempos.

Esta es la razón por la que la Educación Permanente se ha convertido, en los últimos años, en uno de los objetivos prioritarios de las políticas socioeducativas y socioeconómicas en Europa. Sin entrar en un análisis exhaustivo de las causas desencadenantes de este fenómeno, hemos de reconocer que, sin duda, ésta es una de las cuestiones que más espacio ocupan, a diferentes niveles, en la actuación y debate interno de la Unión Europea (programas, presupuesto y financiación, beneficiarios, instituciones, etc.).

Como señala acertadamente la comunicación de la Comisión Europea "Hacer realidad un Espacio Europeo del aprendizaje permanente" (2001), la sociedad del conocimiento, que corre en paralelo con otras tendencias de carácter socioeconómico de las que hablaremos someramente a continuación (nuevas realidades sociolaborales, cambios demográficos, globalización, transformaciones en la estructura familiar, así como el impacto de las tecnologías de la sociedad de la información), plantea a la Unión Europea y a sus ciudadanos tantos beneficios potenciales como retos.

Estos cambios de los que hablamos conforman unas señas de identidad radicalmente diferentes de las de hace muy pocas décadas, entre las que podríamos destacar (López Noguero, 2001):

- Una realidad sociolaboral, amparada en el neoliberalismo, que ha traído consigo un desempleo de carácter estructural. Esta situación tiene su origen en multitud de causas, pero, entre otras razones, y de forma paradójica, se debe al crecimiento económico en alza que hemos presenciado en estos últimos años, con conse- 
cuencias que no se escapan a nadie (fusiones de empresas, reconversiones, etc.). - Una revolución tecnológica que ha acarreado multitud de cambios en las formas de entender el trabajo.

- La definitiva invasión en todos los órdenes de la vida por parte de los medios de comunicación y las nuevas tecnologías. Este fenómeno conlleva una evidente quiebra en las redes tradicionales de comunicación, así como un paulatino aislamiento personal y social, etc.

A la luz de esta realidad, comprobamos cómo los procesos de democratización y de organización social existentes hasta la fecha en la Unión se han visto condicionados e influenciados por la implantación de un modelo socioeconómico de corte neoliberal y de una globalización financiera, apoyado a su vez por acelerados avances tecnológicos. Es evidente que estos factores han subordinado y coartado procesos políticos, sociales, económicos, etc. en la UE (López Noguero, 2005) y, cómo no, políticas educativas que han ido evolucionando de forma radical en las últimas décadas, a los que no ha sido ajena la propia evolución de la Educación Permanente.

En este sentido, la UE en sus comienzos, en los años cincuenta, concedió una mínima, cuando no inexistente, importancia a la educación y a la formación profesional en el marco de los derechos de libre circulación y las políticas sociales europeas, aumentando su peso paulatinamente, como podemos comprobar en la propia evolución de la Educación Permanente en la Unión, que podemos dividir en cuatro etapas fundamentales y que pasamos a desarrollar a continuación:

- Primera etapa (1957-1975).

- Segunda etapa (1976-1991).

- Tercera etapa (1992-1995).

- Cuarta etapa (1996 a nuestros días).

\section{Primera etapa (1957-1975). El "desprecio" de la Educación Permanente}

En la firma del Tratado de Roma, donde se constituía la Comunidad Económica Europea (CEE, precedente inmediato de la Unión), no se manifestaban excesivas preocupaciones por la educación, hecho que posteriormente han lamentado sus propios fundadores ${ }^{1}$. Como afirman, muy acertadamente, Sarrate y Pérez $(2005,47)$, "la educación ha sido la gran ausente en el comienzo de la construcción europea".

No debe extrañarnos la escasa atención a la educación y a la formación en los primeros momentos de la andadura de la Comunidad Europea. Los orígenes comerciales de la misma desplazaron una política educativa a nivel europeo, que sólo empezaría a vislumbrarse hacia los años setenta. En este sentido, debemos hacer constar que la educación no estaba ni siquiera abordada tangencialmente en los documentos iniciales de creación de la Comunidad Europea.

En este sentido, Bayona (1989) afirma que "el hecho de que la educación, como tal, no aparezca en el Tratado de Roma hizo que desde 1957, fecha de su aprobación, hasta 1971 la educación permaneciera como un tema tabú en la CEE". Esta expresión de "tema tabú" para referirse al tratamiento que la UE ha dado históricamente a la educación, ha sido utilizada recurrentemente por diversos autores.

Las razones que explican esta poca atención a la educación por parte de la UE quedan muy razonadas por Valle $(2006,15)$ al señalar, entre otras:

- El carácter, en principio exclusivamente económico, de los Tratados de París (1951) y Roma (1957) que dan origen a la Unión Europea.

- La resistencia de los Estados firmantes de los Tratados a ceder parte de su soberanía, especialmente en una cuestión tan sensible como la educación, íntimamente ligada al concepto de identidad nacional. 
- La dificultad de cristalizar, en ese momento histórico, acciones conjuntas en un terreno como la educación, que presentaba una gran diversidad en los distintos países de la CEE. - La variedad de estructuras político-administrativas que en materia educativa presentaban los países de la CEE, etc.

En este sentido, el propio autor (2006, 15-17) reconoce que el Tratado de París (1951) no realiza ninguna mención a la educación, pero sí advierte que el Tratado de la Comunidad Europea (1957) presenta "algunos apuntes relativos a educación en sentido amplio -más concretamente referidos a la Formación Profesional".

Estos apuntes de los que hablamos se centran en tres artículos del Tratado de Roma (Sarrate, 2000): el 57, que hace referencia al reconocimiento mutuo de diplomas y títulos; el 118, que contempla una referencia al perfeccionamiento profesional, y el 128, que se encomienda al Consejo para que establezca unos principios para la actuación común en formación profesional. Como señala la autora, este marco legal pone en evidencia una especial preocupación comunitaria por la capacitación de los recursos humanos, enfoque que va a prevalecer durante largo tiempo.

Un ejemplo claro de esta poca atención eurocomunitaria a la educación lo podemos comprobar en el hecho de que la primera reunión formal de Ministros de Educación de los países miembros de la Comunidad Europea tuvo lugar en 1971; hasta ese momento no se había pensado en la educación como elemento clave en la construcción europea, y no cabe duda de que dicho año puede considerarse como punto de arranque en el planteamiento de las cuestiones sobre el ámbito educativo en la UE.

Este punto de inflexión tuvo como hito principal el Informe Janne ${ }^{2}$, presentado en febrero de 1973, que ponía de relieve los elementos a utilizar en un futuro para una política comunitaria en materia de educación y, entre ellos, resaltaba como prioritaria la Educación Permanente, reconocida "como factor de transformación de la educación en su conjunto" (Sanz, 2002, 81).

Resumiendo, los escasos esfuerzos eurocomunitarios en esta parcela se centraron en estos años en la formación profesional y formación continua de las empresas, tratando de paliar las necesidades básicas y tecnológicas que tenía la sociedad en esos momentos. Sin embargo, con el devenir de los años siguientes, los Estados miembros fueron considerando cada vez más la educación como un factor esencial para hacer surgir la conciencia comunitaria europea.

\section{Segunda etapa (1975-1991). El nacimiento de la cooperación europea en materia de Educación Permanente}

Tras esta primera fase de diagnóstico previo y concienciación, llegó una mayor cooperación en la política educativa entre los Estados miembros, como lo demuestra el Programa de acción en materia de educación (1976), que, junto a medidas relativas a la atención escolar de los hijos de los trabajadores inmigrantes y a un mayor intercambio de información entre países, preveía también mejorar la enseñanza de las lenguas extranjeras y el aumento de la colaboración en materia de enseñanza universitaria, estableciendo para ello un programa de estudios, investigación, visitas educativas, documentación y estadísticas.

De igual forma, aparece en este año por primera vez la idea de "dimensión europea" asociada a la educación, en la Resolución del Consejo y de los Ministros de Educación de la Comunidad de 9 de febrero de 1976. En este sentido, este concepto de "dimensión europea de la educación" al que se hace referencia de forma tangencial se acabará considerando como un aspecto urgente y necesario en las políticas comunitarias ya en el Acta Única de 1987 (Toranzo, 1998). 
Así, tratando de conseguir una Europa de los ciudadanos, la cooperación entre los Estados miembros en materia de educación fue mucho más intensa a partir de mediados de los ochenta y ha tenido una expresión concreta en programas educativos europeos de todo tipo, aunque con su ámbito principal en lo social, la formación de carácter profesional y la educación universitaria (Ríos, 2004, 206).

Este nuevo enfoque de cooperación educativa a nivel europeo tenía por objeto responder a los nuevos problemas y demandas sociales que se habían generado por la crisis económica de los años setenta. Ejemplo palmario de esta novedosa perspectiva es la ardua obtención de una interpretación amplia, por parte de la CEE, de los escasos artículos del Tratado de Roma que tocasen, aunque fuera tangencialmente, la educación (Sarrate y Pérez, 2005).

En este sentido, Toranzo (1998) y Sanz (2002) destacan, entre otros, los siguientes programas europeos que vieron la luz en estos años y que trataban de dar a la educación y a la formación una nueva dimensión supranacional:

- COMETT (1986): de cooperación entre la Universidad y la empresa en materia de formación en el campo de la tecnología, que contó con dos fases (1986 y 1989).

- IRIS (1987): red europea de programa de formación para la mujer.

- ERASMUS (1987): programa de acción comunitaria en materia de movilidad de estudiantes universitarios.

- PETRA (1988): programa para la formación y la preparación para la transición de los jóvenes a la vida adulta y profesional, mediante la formación profesional inicial y el intercambio de jóvenes trabajadores. - HELIOS (1988): programa destinado a discapacitados.

- NOW (1990): programa de igualdad.

- EUROFORM (1990): programa para la formación en cualificación y competencias profesionales.

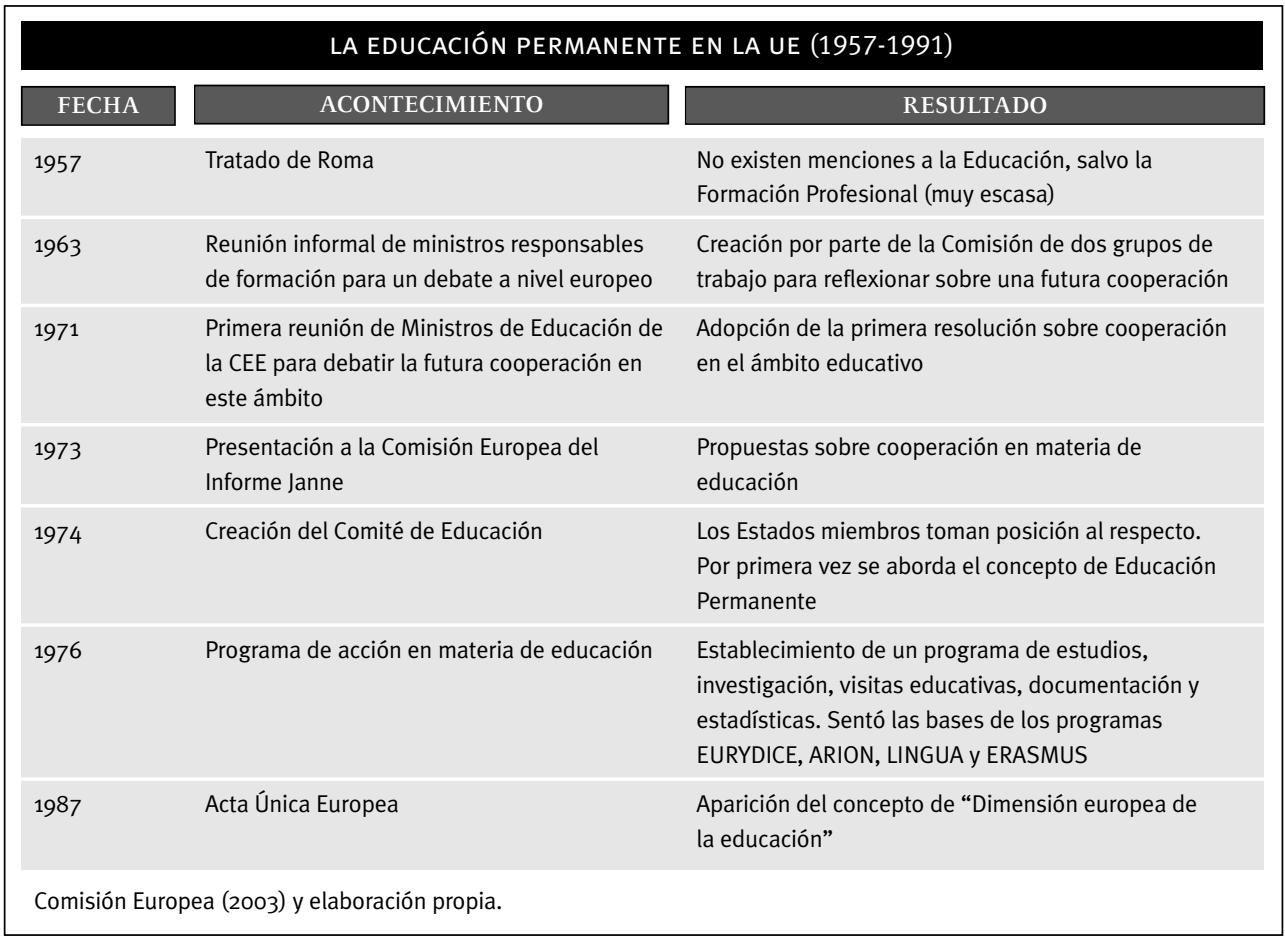


- FORCE (1990): programa de acción para el desarrollo de la formación profesional continua.

- HORIZON (1990): iniciativa comunitaria de empleo y desarrollo de los recursos humanos, especialmente dirigida a personas minusválidas y otros colectivos desfavorecidos. - LEADER (1991): programa para el desarrollo de comarcas rurales.

\section{Tercera etapa (1992-1995). La "dimensión europea" de la educación}

En esta corta pero intensa etapa, se sientan las bases para entender la ampliación que se ha producido en estos años del concepto de Educación Permanente en la UE. Así, debemos resaltar que en esta fase queda consolidada la tendencia de construcción europea en materia de educación que hemos apuntado en la etapa anterior, y un ejemplo claro de ello es la inclusión de la dimensión educativa en el Tratado de la Unión Europea (Tratado de Maastricht), haciendo especial hincapié en la Educación Permanente y de adultos, ya que, como señala Sanz (2002: 83):

"Por primera vez se introducen dos artículos (126 y 127) que hacen referencia explícita y diferenciada a la educación y a la formación. Mientras que en el artículo sobre educación no se hace ninguna referencia a la Educación de Adultos ni a la educación permanente, en el artículo 127 sí se dice claramente que la comunidad se encaminará a mejorar la formación profesional inicial y permanente, para facilitar la inserción y reinserción profesional en el mercado laboral".

Y continúa el autor haciendo una salvedad muy interesante, ya que expone que la UE concede, efectivamente, una importancia especial a la Educación de Adultos en el marco de la formación profesional, pero sin reducir su intervención formativa con adultos exclusivamente a este campo, como sí se había planteado en otras ocasiones en la propia Unión.

Esta inclusión en el Tratado de Maastricht significó un avance importante para la Educación Permanente en la Unión, ya que sentó las bases de las políticas educativas europeas, especialmente en lo que respecta al desarrollo de una educación de calidad y a la política de formación profesional (Sarrate y Pérez, 2005).

Las citadas disposiciones normativas se basan en el principio de subsidiariedad y atribuyen la responsabilidad de organización de los sistemas de educación y de formación profesional a los Estados miembros, limitándose la Unión al fomento de la cooperación de los Estados en materia educativa, al apoyo y complemento de las medidas nacionales en el ámbito de la educación y la formación profesional, al fomento de la cooperación con terceros estados, etc.

Si en un principio se entendía la Educación Permanente como formación profesional y continua, a partir de estos años pasa a ser en la Unión un proceso de aprendizaje que se alarga durante toda la vida, en el que la educación hace frente y se adapta a los cambios de los individuos y las sociedades.

En esta época (1994) se aprueban las iniciativas comunitarias, que favorecían aquellas acciones transnacionales en las que la educación se consideraba elemento fundamental (manteniendo programas existentes, como HORIZON o NOW, y creando otros nuevos como YOUTHSTART, ADAPT, etc.), y también se inician (1995) los dos programas más significativos para el desarrollo de la educación en Europa (SÓCRATES -centrado en potenciar una educación de calidad-y LEONARDO DA VINCI -destinado a la formación profesional inicial y continua-) en un intento de reestructurar los programas europeos de carácter educativo, con el fin de racionalizar las actuaciones comunitarias en materia de educación (Sarrate y Pérez, 2005).

Ambos programas nacieron de la inclusión de los ya citados artículos 126 y 127 del 
Tratado de la Unión Europea de Maastricht, que proporcionaron al Parlamento Europeo y al Consejo las bases para la creación de los dos grandes programas europeos de educación de estos años, tratando de racionalizar las acciones anteriores en materia de educación y formación.

Tanto el programa LEONARDO DA VINCI como el SÓCRATES venían caracterizados por los siguientes rasgos:

- Dimensión europea: que consiste en la introducción de esta idea en la educación, consolidando el sentido de identidad europea, la toma de las ventajas y retos de la construcción europea, el conocimiento de los Estados miembros, etc. ${ }^{3}$

- Valor añadido: ya que la cooperación en materia de educación aportaría un valor añadido real a las acciones individuales de los Estados miembros.

- Respeto a las políticas educativas: puesto que, en última instancia, se reconoce la responsabilidad de los Estados miembros en lo que respecta al contenido y la organización de sus sistemas educativos ${ }^{4}$.

- Construcción de una nueva sociedad: ya que, con programas de este estilo, se buscaban respuestas eficaces que permitieran a los ciudadanos europeos enfrentarse a los cambios sociales, fomentando la capacidad para adaptarse y amoldarse a los cambios económicos y sociales.

- Hacer frente a las desigualdades: fomentando la igualdad de oportunidades entre sexos, garantizando la participación de niños y adolescentes discapacitados, la promoción del acceso a las personas más desfavorecidas, la lucha contra la exclusión social, etc. (Toranzo, 1998: 6-7).

De igual forma, en esta etapa ven la luz dos Libros Blancos que contribuyen decisivamente a esta nueva concepción de Educación Permanente:

- Libro Blanco. Crecimiento, Competitividad y Empleo. Retos y pistas para entrar en el siglo XXI (1993). Este documento hace ver la urgente necesidad que tiene la UE de realizar una inversión inmaterial, en educación y formación. El informe hace ver la conveniencia de buscar el desarrollo, la generalización y la sistematización de la Educación Permanente y formación continua, para mejorar la calidad de la enseñanza, sobre todo técnica, así como el perfeccionamiento profesional. En este Libro Blanco se otorga un protagonismo fundamental al "capital inmaterial" que engloba la educación, las cualificaciones, la aptitud para la innovación y las tradiciones y, desde sus posicionamientos, resalta el papel trascendental que una sociedad que exige la reconstrucción continua del conocimiento debe concederle a la formación en recursos humanos (Sarrate y Pérez, 2005, 47).

- Libro Blanco sobre la Educación y la Formación. Enseñar y aprender. Hacia una sociedad cognitiva (1995), que estudia la situación de la educación en Europa y proclama una serie de orientaciones en el ámbito educativo y técnico profesional. Este documento, que no es sino una muestra más de la preocupación que la Unión iba teniendo por este tema, aboga por luchar contra la exclusión desde instancias socioeducativas, acercar la escuela a la empresa, fomentar la adquisición de nuevos conocimientos, etc. (Ríos, 2004, 206-211) y, como señala la misma autora, "este segundo Libro Blanco trata de abrirnos la mente sobre los cambios que deben efectuarse en la Unión Europea para adquirir la integración social y el desarrollo personal en Europa, por medio de la educación y la formación permanentes". Este Libro Blanco defiende que la UE debe conceder la misma importancia a la inversión en equipamiento que en formación, ya que "la inversión en inteligencia desempeña en efecto un papel determinante para el empleo, la competitividad económica y la cohesión de la sociedad" (Comisión Europea, 1995, 2). 


\section{Cuarta etapa (1996 a nuestros días). La construcción de un Espacio Europeo de aprendizaje para toda la vida}

A nuestro juicio, el hito más importante hasta la fecha en materia de Educación Permanente en la Unión Europea tuvo lugar en 1996 con la proclamación del "Año Europeo de la Educación y la Formación Permanente”, ya que, como apuntan Sarrate y Pérez $(2005,47)$, "por primera vez, se dedica un año europeo a la educación y, específicamente, a la educación de personas adultas, lo que supone otorgar un reconocimiento manifiesto a este ámbito".

Este Año Europeo nació con el fin de hacer conscientes a los europeos de la importancia de la educación y la formación a lo largo de la vida en el desarrollo de la persona, su integración en la vida activa y en la sociedad, así como su participación en el proceso de decisión democrática y su capacidad de adaptación a las transformaciones sociales, económicas, tecnológicas, etc. (Sanz, 2002, 91).

De igual forma, la proclamación de este "Año Europeo de la Educación" pretendía fomentar una mayor cooperación entre las estructuras educativas y de formación y las empresas, así como contribuir a la realización de un espacio europeo de la educación y la formación mediante el reconocimiento académico y profesional de las cualificaciones en la Unión Europea y destacar la contribución de la educación y la formación a la igualdad de oportunidades 5 .

A lo largo del citado Año Europeo se llevaron a cabo acciones de información, sensibilización y promoción de la educación y la formación permanentes (incluyendo manifestaciones de carácter general o temáticas, la elaboración y difusión de productos de comunicación, publicaciones, conferencias, seminarios, así como estudios y sondeos), abordando temas tales como la importancia de una educación general de alta calidad, la motivación de las personas para acceder a la educación y a la Formación Permanentes, el fomento de una mayor cooperación entre los centros de enseñanza y formación y los medios económicos, el desarrollo de la dimensión europea de la educación y de la formación iniciales y continua, la sensibilización de los interlocutores sociales y de los padres, el fomento de una formación profesional que culmine en cualificaciones concretas, etc.

La proclamación de este Año Europeo tuvo un impacto muy destacable en el seno de la UE y consiguió que la Educación y la Formación Permanentes ocupasen un lugar importante en la reflexión política. Gracias a él, nuevos agentes participaron en un ámbito que anteriormente estaba reservado a los especialistas en la materia. Además, los responsables de varios Estados miembros señalaron que el Año Europeo había abierto nuevas posibilidades de mayor colaboración entre los ministerios o los diversos niveles administrativos.

En 2001, tratando de hacer frente a la transición hacia una sociedad del conocimiento, la Comisión Europea apoyó la aplicación de estrategias de aprendizaje permanente, así como de acciones concretas, con el fin de crear un espacio europeo del aprendizaje permanente, en la Comunicación de la Comisión de 21/11/2001 "Hacer realidad un espacio Europeo del aprendizaje permanente" .

Esta Comunicación de la Comisión comienza a trabajarse cuando el Consejo Europeo de Feira de junio de 2000 solicitó a la Comisión (y a los Estados miembros) que definiesen una estrategia coherente capaz de dar acceso a todos los europeos a ofertas de aprendizaje permanente, que puso en marcha una amplia consulta a escala europea $^{7}$.

La Comisión Europea señala en este documento que el futuro de Europa depende de la medida en que sus ciudadanos puedan afrontar los retos económicos y sociales, y se piensa que un "espacio europeo del apren- 
dizaje permanente" les permitirá pasar con libertad de un entorno de aprendizaje a un entorno de trabajo y de una región a otra o de un país a otro para utilizar de la mejor manera posible sus aptitudes y sus cualificaciones.

La Comunicación de 2001 establece los componentes de esas estrategias para contribuir al esfuerzo de los Estados miembros y otros agentes. En este sentido, la transformación de los sistemas tradicionales debe ser, según esta Comunicación, la primera etapa para que todos los ciudadanos puedan tener acceso a las ofertas de aprendizaje permanente, entendido como todo aquel aprendizaje que va desde la enseñanza preescolar hasta después de la jubilación, en lo que gráficamente queda apuntado como aprendizaje "desde la cuna hasta la tumba" y que, al mismo tiempo, comprende todas las formas de educación (formal, informal o no formal).

Al hilo de esta Comunicación de la Comisión, en el Consejo Europeo de Lisboa de marzo de 2000 quedan enunciados tanto el objetivo estratégico educativo de lograr que la UE se convierta en la economía basada en el conocimiento más competitiva y dinámica del mundo, como el marco de cooperación política integrado denominado "Educación y Formación 2010", que cristaliza en una redacción definitiva en $2003^{8}$ y que tenía por principio director el aprendizaje permanente, en sinergia con los elementos pertinentes de las políticas de juventud, empleo, inclusión social e investigación, que se ha dado en llamar "Estrategia de Lisboa"9.

En este sentido, las nuevas directrices integradas de la UE, adoptadas en el marco de la referida Estrategia de Lisboa, preveían también la consecución del objetivo del aprendizaje permanente y de favorecer el crecimiento y la cohesión social mediante la educación y la formación de los ciudadanos (Pérez Serrano, 2006); de hecho, los sucesivos Consejos Europeos, desde el de Feira del año 2000 en adelante, hicieron hincapié en la necesidad de aplicar estrategias coherentes y globales de aprendizaje permanente, comprometiéndose todos los Estados miembros a aplicar tales estrategias con vistas a un futuro cercano.

Como colofón a estos precedentes, en noviembre de 2006, el Parlamento Europeo y el Consejo establecen un Programa de Aprendizaje Permanente (2007-2013), que trata de desarrollar y reforzar los intercambios, la cooperación y la movilidad para que los sistemas de educación y formación dentro de la Comunidad se conviertan en una referencia de calidad mundial con arreglo a la Estrategia de Lisboa y poder contribuir al desarrollo de la UE como sociedad del conocimiento avanzada, caracterizada por un desarrollo económico sostenible, con más y mejores empleos y con mayor cohesión social ${ }^{10}$.

Los objetivos específicos de este programa son los siguientes: contribuir al desarrollo de una educación y una formación de calidad; promover la innovación y la dimensión europea en los sistemas y las prácticas vigentes; apoyar la realización de un Espacio Europeo del Aprendizaje Permanente; ayudar a mejorar la calidad, el atractivo y la accesibilidad de las oportunidades de educación y formación; reforzar la cohesión social, la ciudadanía activa, el diálogo intercultural, la igualdad entre hombres y mujeres y la realización personal; favorecer una mayor participación de personas de todas las edades, incluidas las que tienen necesidades especiales y las pertenecientes a grupos desfavorecidos, etc.

Por lo que respecta a su organización, debemos señalar que incluye todo el conjunto de los programas europeos anteriores en el ámbito de las políticas educativas de la Unión (SÓCRATES, LEONARDO DA VINCI, E-LEARNING, la acción JEAN MONNET, etc.), que se basa en la estructura anterior del periodo 2000-2006 y se encuentra subdividido en seis subprogramas, cuatro de los cuales son programas sectoriales, todos ellos estructurados de forma similar. De igual 
forma, debemos señalar que todas las acciones integran la movilidad, las lenguas y las nuevas tecnologías.

La Comisión ha decidido constituir el programa con esta estructura porque se entiende que un sistema único de ayuda comunitaria en el ámbito de la educación y la formación supone mayor racionalidad, coherencia y eficacia. De igual forma, se entiende que así debería permitir una mayor interacción entre los distintos ámbitos y una mayor visibilidad, sobre todo por lo que se refiere a la capacidad para responder a los cambios experimentados en este ámbito y a una mayor cooperación. En este sentido, los subprogramas a los que hacíamos referencia son los siguientes:

- COMENIUS: cuyo objeto es la enseñanza preescolar y escolar hasta el final del segundo ciclo de enseñanza secundaria.

- ERASMUS: centrado en la educación superior formal y la formación profesional avanzada, sea cual sea la duración de la carrera o cualificación, incluidos los estudios de doctorado. A diferencia de los programas anteriores, la formación profesional avanzada ya no está incluida en LEONARDO DA VINCI, sino en ERASMUS. - LEONARDO DA VINCI: que gira alrededor de la formación profesional que no sea de nivel superior.

- GRUNDTVIG: referido a todo tipo de educación para adultos ${ }^{11}$.

- JEAN MONNET: aborda específicamente las cuestiones relativas a la integración europea en el ámbito universitario y el apoyo a los centros y asociaciones activos en materia de educación y formación a escala europea.

- TRANSVERSAL: que se refiere a actividades concretas que superan los límites de los programas sectoriales.

En los últimos años, la UE ha hecho especial énfasis en la Educación de Adultos, como lo demuestra la Comunicación de la Co- misión "Aprendizaje de adultos: nunca es demasiado tarde para aprender" (2006), inscrita en el marco de la Estrategia de Lisboa. En la citada Comunicación se señala que, aunque los Estados miembros reconocen la importancia del aprendizaje permanente, hoy día, en Europa, el número de alumnos adultos sigue por debajo del objetivo fijado por los Estados miembros. A través de la citada Comunicación, la Comisión alienta a los Estados miembros a velar por la calidad de su sistema de aprendizaje de adultos y por el nivel general de aptitud de sus ciudadanos ${ }^{12}$.

En esta Comunicación, la Comisión señala los siguientes retos sobre la Educación Permanente y de Adultos:

- Competitividad económica: a juicio de la Comisión, se debe aumentar el nivel general de aptitud de los ciudadanos de todos los Estados miembros, ya que ésta es una cuestión económica trascendental, que contribuye a alcanzar los objetivos de crecimiento, empleo y cohesión social fijados por la Estrategia de Lisboa.

- Cambios demográficos: a juicio de la Comisión, los sistemas de aprendizaje deben tomar en consideración el envejecimiento de la población europea y el papel cada vez más importante de la población inmigrante.

- Pobreza y exclusión social: el trabajo educativo con adultos puede desempeñar un papel clave en la lucha contra la pobreza y la exclusión social, que marginan a un número significativo de personas en todos los Estados miembros.

En resumen, a juicio de la UE, aumentar los niveles generales de aptitud de los ciudadanos significa mejorar sus oportunidades profesionales y contribuir a la lucha contra la pobreza y la exclusión social. Para alcanzar este objetivo, mediante esta Comunicación que analiza la cuestión del aprendizaje de adultos, la Comisión estimula a los Estados miembros para que multipliquen y consoli- 
den las oportunidades de aprendizaje para adultos y las hagan accesibles a todos los ciudadanos.

\section{Conclusiones}

La función básica de la educación es conseguir que la persona se socialice, es decir, que se integre de manera efectiva en la sociedad que le ha tocado vivir, y, por ello, el hecho educativo no es, o al menos no debería serlo, un proceso estático.

Todo lo contrario, si entendemos que la educación debe "promover la transmisión y la apropiación de aquellos saberes que la sociedad exige en un momento determinado para incluirse, para que los derechos a la integración realmente se efectivicen, para que los sujetos gocen de una ciudadanía plena" (Fernández, 2001, 319), ésta debería evolucionar constantemente, adaptándose a las transformaciones sociales que vayan aparecien- do, para garantizar la realización personal, facilitar la incorporación a la vida activa y promover el sentido de la participación en la sociedad.

Desde la proclamación del Año Europeo de la Educación y la Formación Permanentes en 1996, en el que se fomentó en el seno de la UE el concepto de aprendizaje durante toda la vida, sensibilizando a la población en general acerca de la necesidad y la conveniencia de seguir aprendiendo y estimulando el debate sobre la forma en que los sistemas de formación pueden dar respuesta satisfactoria a las nuevas necesidades de aprendizaje, la Educación Permanente ha cambiado radicalmente en Europa.

La sociedad europea está cambiando a pasos acelerados y sus ciudadanos se deberán enfrentar, en un futuro más o menos cercano, a situaciones y problemas que aún hoy desconocemos, implicando cambios de mentalidad y cultura para los que muy pocos están aún preparados (Pérez Pérez, 2006); por

\begin{tabular}{|c|c|c|}
\hline FECHA & ACONTECIMIENTO & RESULTADO \\
\hline 1992 & Tratado de Maastrich & Se incorpora la educación a los Tratados de la UE \\
\hline 1993 & $\begin{array}{l}\text { Libro Blanco. Crecimiento, competitividad y } \\
\text { Empleo }\end{array}$ & $\begin{array}{l}\text { Se sitúa la educación y la formación en el núcleo } \\
\text { de las políticas económicas, sociales y de empleo }\end{array}$ \\
\hline 1995 & $\begin{array}{l}\text { Aparición de los Programas Sócrates y } \\
\text { Leonardo }\end{array}$ & $\begin{array}{l}\text { Programas que reúnen y desarrollan programas } \\
\text { previamente existentes en un programa global de } \\
\text { acción en materia educativa, al objeto de crear una } \\
\text { “Europa del conocimiento" y reforzar la “dimensión } \\
\text { europea” de la educación }\end{array}$ \\
\hline 1996 & $\begin{array}{l}\text { Proclamación del “Año Europeo de la } \\
\text { Educación y la Formación Permanentes” }\end{array}$ & $\begin{array}{l}\text { La educación y la formación permanentes ocupan un } \\
\text { lugar importante en la reflexión política de la UE }\end{array}$ \\
\hline 2000 & Consejo Europeo de Lisboa & $\begin{array}{l}\text { Se fija el objetivo de conseguir, para 2010, la } \\
\text { economía, basada en el conocimiento, más competitiva } \\
\text { y dinámica del mundo. Para conseguir ese objetivo se } \\
\text { apoyará en el aprendizaje permanente }\end{array}$ \\
\hline 2001 & $\begin{array}{l}\text { Comunicación de la Comisión “Hacer realidad } \\
\text { un espacio Europeo del aprendizaje permanente" }\end{array}$ & $\begin{array}{l}\text { Aplicación de estrategias de aprendizaje permanente } \\
\text { a las políticas e la Unión }\end{array}$ \\
\hline 2006 & $\begin{array}{l}\text { Programa de Aprendizaje Permanente } \\
(2007-2013)\end{array}$ & $\begin{array}{l}\text { Creación de un Programa único en materia } \\
\text { educativa en la UE }\end{array}$ \\
\hline
\end{tabular}


todo ello, el debate de la Educación Permanente sigue abierto.

En este sentido, la contribución de la Unión al debate mundial sobre la Educación y la Formación Permanentes pivota alrededor de un enfoque amplio de "acompañamiento a lo largo de toda la vida", que no hace depender el aprendizaje de imperativos económicos y reconoce el derecho al enriquecimiento personal, la participación en el proceso democrático de toma de decisiones, el aprendizaje por propio interés y el envejecimiento activo. Todos éstos son conceptos claves para entender la Educación Permanente hoy, preparando a una sociedad, la europea, que se caracteriza cada vez más por la diversidad cultural y linguística.

Como señala Sarrate (2000, 259), se ha producido en el marco de la Unión un creciente interés por la Educación Permanente y "la evolución en este sentido ha sido verdaderamente importante, pues si una de las primeras inquietudes en materia educativa de la Unión ha sido la formación profesional, en nuestros días el centro de atención recae en la educación a lo largo de la vida, principio educativo que día a día va retomando la tradición humanista y democrática que la debe caracterizar".

No obstante, tampoco podemos perder de vista que un mejor aprendizaje de adultos también puede jugar un papel clave en la formación profesional de los ciudadanos europeos y en la inserción social de los grupos más desfavorecidos en el mercado laboral, como los inmigrantes y las personas de más edad, cada vez más numerosos en Europa. Además, la mejora del aprendizaje de adultos representa una ventaja considerable tanto en el plano colectivo como en el individual.

En cualquier caso, el debate sigue abierto, continuamos trabajando en pos de una Educación Permanente en la UE, siempre en clave de equidad y eficiencia, donde deberán tener una importancia clave la planificación a largo plazo a nivel local y nacional a la hora de definir las prioridades, la validación del aprendizaje en todos los contextos que permitan la adquisición de conocimientos o competencias esenciales, así como apoyar la eficiencia de la política y políticas transectoriales de la Unión.

\section{Referencias bibliográficas}

Comisión Europea (1993): Libro Blanco. Crecimiento, Competitividad y Empleo. Retos y pistas para entrar en el siglo XXI. Bruselas: Oficina de Publicaciones Oficiales de las Comunidades Europeas.

Comisión Europea (1995): Libro Blanco sobre la educación y la formación. Enseñar y aprender. Hacia una sociedad cognitiva. Bruselas: Oficina de Publicaciones Oficiales de las Comunidades Europeas.

Comisión Europea (2001): Comunicación de la Comisión. Hacer realidad un Espacio Europeo del aprendizaje permanente. Bruselas: Comisión Europea.

Comisión Europea (2003): Europa paso a paso. Cooperación en el ámbito de la enseñanza superior. Luxemburgo: Oficina de Publicaciones oficiales de las Comunidades Europeas.

Comisión Europea (2006): Comunicación de la Comisión. Aprendizaje de adultos: nunca es demasiado tarde para aprender. Bruselas: Comisión Europea.

Fernández, M. C. (2001): "Educación Social y ciudadanía”. Pedagogía Social. Revista Interuniversitaria de Pedagogía Social, 6-7, pp. 307-319.

López Noguero, F. (2001): "Prólogo", en López Noguero, F.; Cruz, R., y Beltrán, R.: Formación Permanente: tendencias actuales. Huelva: Educación.

López Noguero, F. (2005): “La Educación Popular en España, retos e interrogantes”. @gora digital. Revista electrónica, 6. www.uhu.es/agora.

Pérez Pérez, I. (2006): “Metodología participativa en la enseñanza universitaria”. Revista Interuniversitaria de Pedagogía Social. 14, pp. 160-161.

Pérez Serrano, G. (2006): "Presentación”. Revista Interuniversitaria de Pedagogía Social. 14, pp. 5-8.

Ríos González, M. (2004): “Evolución de la Educación Permanente en Europa”. Eúphoros, 7, pp. 203-214. 
Sanz Fernández, F. (2002): “Génesis y desarrollo de la educación de personas adultas”, en López-Barajas, E. y Sarrate Capdevila, Mํㅡㄹ L.: La educación de personas adultas: reto de nuestro tiempo. Madrid: Dykinson.

Sarrate Capdevila, Mํㅡㄹ L. (2000): "La educación a lo largo de toda la vida, prioridad de la Unión Europea". Revista de Educación XXI, Facultad de Educación de la UNED, 3, pp. 245-262.

Sarrate Capdevila, Mํㅡㄹ. y Pérez de Guzmán Puya, M. V. (2005): "Educación de personas adultas. Situación actual y propuestas de futuro". Revista de Educación, 336, pp. 41-57.

Toranzo López, F. J. (1998): Programas europeos de educación. Carmona (Sevilla): Junta de Andalucía.

Valle, J. M. (2006): La Unión Europea y su política educativa (medio siglo de acciones en materia de educación). Madrid: CIDE.

\section{Notas}

${ }^{1}$ Jean Monnet, uno de los padres de la UE y que jugó en los años cincuenta un papel decisivo en la concepción de este instrumento de integración continental, llegó a afirmar al respecto que "si hubiera que empezar de nuevo (la construcción europea), empezaría por la educación".

${ }^{2}$ Encargado al antiguo ministro belga de educación M. Janne.

${ }^{3}$ Desde estos momentos, esta dimensión europea aparecerá desde el carácter transnacional de los proyectos y en la consecución de un espacio europeo de actividades.

${ }^{4}$ Con ello se excluyó toda armonización de las disposiciones legales y reglamentarias de los diferentes Estados.

${ }^{5}$ Decisión n ${ }^{\text {o }}$ 95/2493/CE del Parlamento Europeo y del Consejo, de 23 de octubre de 1995, por la que se declara 1996 como el "Año Europeo de la Educación y de la Formación Permanentes".

${ }^{6}$ COM (2001) 678 final (no publicada en el Diario Oficial).

${ }^{7}$ Esta Comunicación es el resultado de esa encuesta y del debate social posterior, en el que participaron entre 2000 y 2001 unas 12.000 personas, además de los propios Estados miembros, los países del EEE (Espacio Económico Europeo), así como paí- ses candidatos, instituciones comunitarias, interlocutores sociales, ONG, etc.

${ }^{8}$ Comisión Europea (2003): Comunicación de la Comisión. Educación y Formación 2010: Urgen las reformas para coronar con éxito la estrategia de Lisboa. Proyecto de informe intermedio conjunto sobre la aplicación del programa de trabajo detallado relativo al seguimiento de los objetivos de los sistemas de educación y formación en Europa. COM (2003) 685 final (no publicada en el Diario Oficial).

${ }^{9}$ Que fijaba, entre otros, objetivos de crecimiento económico, competitividad e inserción social a cubrir por la UE en un futuro.

${ }^{10}$ Decisión n ${ }^{\mathrm{o}}$ 1720/2006/CE del Parlamento Europeo y del Consejo, de 15 de noviembre de 2006, por la que se establece un programa de acción en el ámbito del aprendizaje permanente (Diario Oficial L 327 de 24/11/2006).

${ }^{11}$ Este programa trata de responder al reto del envejecimiento de la población en Europa en el ámbito de la educación, así como ayudar a que los adultos adquieran medios de mejorar sus conocimientos y competencias.

${ }^{12}$ Comunicación de la Comisión, de 23 de octubre de 2006, "Aprendizaje de adultos, nunca es demasiado tarde para aprender". COM (2006) 614 final (no publicada en el Diario Oficial).

DIRECCIÓN DEL AUTOR: Fernando López Noguero. Universidad Pablo de Olavide. Departamento de Ciencias Sociales. Carretera de Utrera, km.1. 41013 Sevilla.

Correo electrónico: flopnog@upo.es.

Fecha de recepción del artículo: 09.IX.2007

Fecha de aceptación definitiva: 17.X.2007 
